OPEN ACCESS

Edited by:

Claudio Fabricio Gonzalez,

University of Florida, United States

Reviewed by:

Fernando A. Pagliai,

University of Florida, United States

Marcelo Luciano Merli,

National University of Rosario,

Argentina

*Correspondence:

Tino Krell

tino.krell@eez.csic.es

tThese authors have contributed equally to this work.

Specialty section:

This article was submitted to Microbial Physiology and Metabolism,

a section of the journal

Frontiers in Microbiology

Received: 06 April 2017 Accepted: 17 May 2017

Published: 31 May 2017

Citation:

Fernández M, Matilla MA, Ortega Á and Krell T (2017) Metabolic Value Chemoattractants Are Preferentially

Recognized at Broad Ligand Range Chemoreceptor of Pseudomonas putida KT2440.

Front. Microbiol. 8:990 doi: 10.3389/fmicb.2017.00990

\section{Metabolic Value Chemoattractants Are Preferentially Recognized at Broad Ligand Range Chemoreceptor of Pseudomonas putida KT2440}

\author{
Matilde Fernández ${ }^{\dagger}$, Miguel A. Matilla ${ }^{\dagger}$, Álvaro Ortega and Tino Krell* \\ Estación Experimental del Zaidin, Department of Environmental Protection, Consejo Superior de Investigaciones Cientificas,
} Granada, Spain

Bacteria have evolved a wide range of chemoreceptors with different ligand specificities. Typically, chemoreceptors bind ligands with elevated specificity and ligands serve as growth substrates. However, there is a chemoreceptor family that has a broad ligand specificity including many compounds that are not of metabolic value. To advance the understanding of this family, we have used the PcaY_PP (PP2643) chemoreceptor of Pseudomonas putida KT2440 as a model. Using Isothermal Titration Calorimetry we showed here that the recombinant ligand binding domain (LBD) of PcaY_PP recognizes 17 different C6-ring containing carboxylic acids with $K_{D}$ values between 3.7 and $138 \mu \mathrm{M}$ and chemoeffector affinity correlated with the magnitude of the chemotactic response. Mutation of the pcaY_PP gene abolished chemotaxis to these compounds; phenotype that was restored following gene complementation. Growth experiments using PcaY_PP ligands as sole C-sources revealed functional relationships between their metabolic potential and affinity for the chemoreceptor. Thus, only 7 PcaY_PP ligands supported growth and their $K_{D}$ values correlated with the length of the bacterial lag phase. Furthermore, PcaY_PP ligands that did not support growth had significantly higher $K_{D}$ values than those that did. The receptor has thus binds preferentially compounds that serve as C-sources and amongst them those that rapidly promote growth. Tightest binding compounds were quinate, shikimate, 3-dehydroshikimate and protocatechuate, which are at the interception of the biosynthetic shikimate and catabolic quinate pathways. Analytical ultracentrifugation studies showed that ligand free PcaY_PP-LBD is present in a monomer-dimer equilibrium $\left(K_{D}=57.5 \mu \mathrm{M}\right)$. Ligand binding caused a complete shift to the dimeric state, which appears to be a general feature of four-helix bundle LBDs. This study indicates that the metabolic potential of compounds is an important parameter in the molecular recognition by broad ligand range chemoreceptors.

\footnotetext{
Keywords: chemotaxis, chemoreceptor, ligand recognition, isothermal titration calorimetry, Pseudomonas
} 


\section{INTRODUCTION}

Bacteria need to constantly adapt to changing environmental conditions to assure survival. These adaptations are achieved through a variety of signal transduction systems that most commonly include one- and two-component systems as well as chemosensory pathways (Galperin, 2005; Laub and Goulian, 2007; Hazelbauer et al., 2008). In the latter systems, signaling is initiated either by the direct binding of chemoeffectors or chemoeffector-loaded periplasmic binding proteins to the ligand binding domain (LBD) of chemoreceptors (Matilla and Krell, 2017). Chemoreceptor activation triggers a molecular stimulus that modulates CheA autophosphorylation and consequently transphosphorylation of the CheY response regulator, which generates the pathway output (Hazelbauer et al., 2008). Chemosensory pathways were shown to mediate chemotaxis and type IV pili-based motility, or are involved in regulating alternative cellular processes (Hickman et al., 2005; Zusman et al., 2007; Wuichet and Zhulin, 2010).

Chemotaxis provides bacteria with the capacity to approach or escape from different compounds, therefore favoring the movement toward optimal ecological niches. Thus, chemotaxis systems of different bacteria have evolved to facilitate adaptation to specific ecological niches. Escherichia coli is the traditional model to study chemosensory signaling mechanisms. This bacterium has four chemoreceptors and an aerotaxis receptor that feed into a single chemotaxis pathway (Parkinson et al., 2015). However, several alternative model organisms have been established to investigate chemotaxis in bacteria with lifestyles different to that of the enterobacterium E. coli.

One of these models is Pseudomonas putida KT2440 (Belda et al., 2016). This strain was isolated from soil, is nutritionally versatile, has a saprophytic lifestyle, colonizes plant roots efficiently and is able to protect plants against phytopathogens (Bagdasarian et al., 1981; Espinosa-Urgel et al., 2002; Nakazawa, 2002; Regenhardt et al., 2002; Matilla et al., 2010). P. putida KT2440 has 27 different chemoreceptors and data available suggest that receptors feed into three chemosensory pathways that are homologous to the che, wsp, and chp pathways of $P$. aeruginosa (Garcia-Fontana et al., 2013). Information on the function of many chemoreceptors of P. putida KT2440 has been obtained which are summarized in Supplementary Figure 1 and Table 1. Characterized KT2440 chemoreceptors include McpS (Lacal et al., 2010; Pineda-Molina et al., 2012), McpQ (Martin-Mora et al., 2016) and McpR (Parales et al., 2013), for different tricarboxylic acid (TCA) cycle intermediates, McpP for C2- and C3-organic acids (Garcia et al., 2015), McpA for L-amino acids (Corral-Lugo et al., 2016), McpU for polyamines (Corral-Lugo et al., 2016), McpG for gamma-aminobutyrate (Reyes-Darias et al., 2015a) or $\mathrm{McpH}$ for metabolizable purines (Fernandez et al., 2016). In addition, there are three paralogous receptors for energy taxis (Sarand et al., 2008) as well as homologs of the P. aeruginosa WspA and PilJ chemoreceptors, that feed into chemosensory pathways regulating c-di-GMP levels or mediate type IV pili-based motility, respectively
(Hickman et al., 2005; Persat et al., 2015; Corral-Lugo et al., 2016). The remaining 14 chemoreceptors are of unknown function.

The inspection of ligand profiles of chemoreceptors suggests that the primary physiological function of chemotaxis resides in the capacity to approach compounds that are of metabolic value and that can be used for growth. For example, all 34 compounds that were shown to bind and activate the eight characterized KT2440 chemoreceptors mentioned above serve the bacterium either as carbon or nitrogen sources. However, there is evidence for another class of chemoreceptors that is characterized by a very broad ligand range and where only few of the recognized chemoattractants appear to be of metabolic value. One such chemoreceptor is McpT of P. putida DOT-T1E (Lacal et al., 2011), which recognizes and mediates chemotaxis to a wide range of different mono- and biaromatic compounds of which only few can be used as nutrient sources. The physiological sense of the response to the remaining compounds is unclear, but may represent the sensing of certain environmental cues as proposed by Yang et al. (2015).

Another member of this broad ligand range receptor family is PcaY of $P$. putida F1; a chemoreceptor that responds to a number of C6-ring containing carboxylic acids (Luu et al., 2015). PcaY chemoeffectors include, for example, the nonaromatic quinate and shikimate as well as various aromatics like benzoate, 4-hydroxybenzoate, protocatechuate, vanillate and vanillin. Interestingly, Luu et al. (2015) observed weak sequence similarities (16\% identity) between the LBDs of McpT and PcaY. As for the mode of chemoeffector recognition, the authors expected that PcaY binds aromatic acids, but were unable to rule out indirect binding mediated by periplasmic binding proteins. In addition, it could not be ruled out that downstream intermediates are sensed by the chemoreceptor rather than the compounds themselves (Luu et al., 2015). Precedents for such mechanism have recently been reported for the chemoreceptors MCP2201 and MCP2983 of Comamonas testosteroni that mediate chemotaxis to multiple aromatic compounds, including several PcaY effectors such as 4-hydroxybenzoate, vanillate, vanillin, and protocatechuate (Ni et al., 2013a, 2015). The authors showed that none of these compounds bound to the recombinant LBDs of these receptors ( $\mathrm{Ni}$ et al., 2013a, 2015). Instead, different TCA cycle intermediates bound and it was proposed that chemotaxis toward aromatics is caused by the recognition of compounds that are generated during aromatic compound metabolism.

The ORF encoding the chemoreceptor PP2643 of P. putida KT2440 is homologous to PcaY of P. putida F1 (97\% sequence identity) and has therefore been named PcaY_PP. In this study, we used PcaY_PP as a model to study ligand recognition at broad ligand range chemoreceptors. We will define the ligand profile of this receptor, assess whether receptor activation occurs by direct binding or an alternative indirect mechanism and evaluate the relationship between the receptor affinity and metabolic value of the different ligands. Our data show that this chemoreceptor recognizes preferentially ligands that are of metabolic value. A significant number 
of ligands were of no apparent metabolic value and may correspond to environmental cues that are sensed by the bacterium.

\section{EXPERIMENTAL PROCEDURES}

\section{Bacterial Strains, Culture Media, and Growth Conditions}

Bacterial strains used in this study are listed in Table 1. P. putida KT2440 and its derivative strains were routinely grown at $30^{\circ} \mathrm{C}$ in $\mathrm{LB}$ or $\mathrm{M} 9$ medium supplemented with $1 \mathrm{mM} \mathrm{MgSO}_{4}$, $6 \mathrm{mg} \mathrm{l}^{-1}$ Fe-citrate (Abril et al., 1989) and $10 \mathrm{mM}$ sodium benzoate or glucose as carbon source. $P$. putida KT2440R was grown in the same medium supplemented with rifampicin $\left(10 \mu \mathrm{g} \mathrm{ml}^{-1}\right)$. For growth experiments to assess the capacity of the PcaY_PP ligands to support growth a sole C-source, KT2440 cells were pre-cultured overnight in M9 medium supplemented with $10 \mathrm{mM}$ glucose and washed twice with $\mathrm{M} 9$ medium, prior to the inoculation of M9 medium containing $10 \mathrm{mM}$ of the different carbon sources; except for 3-CBA, 4-CBA, 3-NBA, 4-NBA, 3-MBA, and 4-MBA that, due to their lower solubility, were used at $2 \mathrm{mM}$. When necessary, the $\mathrm{pH}$ of the medium was adjusted to 7.0 prior to inoculation. Bacterial growth rates were monitored by measuring the $\mathrm{OD}_{660}$ until cultures reached the stationary phase. The growth rate during the exponential growth $(\mathrm{m})$ was calculated according to $\mathrm{m}=\left(\mathrm{LnOD}_{2}-\mathrm{LnOD}_{1}\right) \cdot\left(\mathrm{t}_{2}-\mathrm{t}_{1}\right)^{-1}$, where $\mathrm{OD}_{2} / \mathrm{t}_{2}$ and $\mathrm{OD}_{1} / \mathrm{t}_{1}$ correspond to the $\mathrm{OD}_{660} /$ time at the end and the beginning of the time interval. The generation time $(G)$ was defined by $G=\operatorname{Ln} 2 \mu^{-1}$. The duration of the lag phase was calculated from the moment of inoculation to the start of the exponential phase as described in Swinnen et al. (2004). Normalized growth yield per carbon source after $24 \mathrm{~h}$ was determined applying correlation factors between cell dry weight and $\mathrm{OD}_{660}$ determined in batch cultures and assuming that all the carbon source was consumed during growth. Data were fitted by linear least squares regression or submitted to the chi-squared test with
Yates correction using in both cases the Graph Pad Prism v7.02 software.

\section{Construction of the Expression Plasmid for PcaY_PP-LBD}

The PcaY_PP-LBD (amino acids 44-196) was defined as the segment between both transmembrane regions as predicted by the DAS transmembrane region prediction algorithm (Cserzo et al., 1997). The corresponding DNA fragment was amplified by PCR using primers 5'-AACATATG GGTAGCGACCAGCAGATCAC-3', containing a NdeI restriction site (underlined), and 5'-AAGGATCCCTACCGG GCCAGACGCCGGTCGGA-3', containing a BamHI site (underlined) as well as a stop codon. The PCR product was cloned into pET28b (Novagen), previously digested with the same enzymes. The ligation mixture was dialyzed and electroporated into E. coli DH5 $\alpha$. Transformants were selected on kanamycin $\left(50 \mu \mathrm{g} \mathrm{ml}^{-1}\right) \mathrm{LB}$ plates and the resulting plasmid pET28-PcaY_PP-LBD was verified by sequencing the insert and flanking regions prior to the transfer into E. coli BL21 (DE3) by electroporation.

\section{Protein Overexpression and Purification}

Escherichia coli strain BL21 (DE3) pET28-PcaY_PP-LBD was grown in 21 Erlenmeyer flasks containing $500 \mathrm{ml} \mathrm{LB}$ supplemented with $50 \mu \mathrm{g} / \mathrm{ml}$ kanamycin at $30^{\circ} \mathrm{C}$ until an $\mathrm{OD}_{660}$ of 0.6 , at which point protein expression was induced by adding $0.5 \mathrm{mM}$ isopropyl-beta-D-thiogalactopyranoside. Growth was continued at $18^{\circ} \mathrm{C}$ overnight and cells were harvested by centrifugation at $10,000 \mathrm{~g}$ for $30 \mathrm{~min}$. Cells were then resuspended in buffer A $(20 \mathrm{mM}$ Tris/ $\mathrm{HCl}, 200 \mathrm{mM} \mathrm{NaCl}$, $10 \mathrm{mM}$ imidazole, $5 \%$ (v/v) glycerol, $0.1 \mathrm{mM}$ EDTA, $\mathrm{pH} 8.0$ ) and broken by French Press treatment. After centrifugation at $20,000 \mathrm{~g}$ for $15 \mathrm{~min}$, the supernatant was loaded onto a $5 \mathrm{ml}$ HisTrap column (Amersham Bioscience), washed with 10 column volumes of buffer $A$ and eluted with an imidazole gradient of $45-500 \mathrm{mM}$ in the same buffer. Protein containing fractions were pooled and dialyzed overnight at $4^{\circ} \mathrm{C}$ into

TABLE 1 | Bacterial strains and plasmids used in this study.

\begin{tabular}{|c|c|c|}
\hline Strain or plasmid & Relevant characteristics ${ }^{a}$ & Reference \\
\hline \multicolumn{3}{|l|}{ Strains } \\
\hline Escherichia coli BL21 (DE3) & $\mathrm{F}^{-}$, ompl, hsd $\mathrm{S}_{\mathrm{B}}\left(\mathrm{r}^{-}{ }_{\mathrm{B}} \mathrm{m}^{-} \mathrm{B}\right) \mathrm{gal}$, dam, met & Jeong et al., 2009 \\
\hline E. coli $\mathrm{DH} 5 \alpha$ & supE44 lacU169 (Ø80lacZ $\Delta \mathrm{M} 15)$ hsdR17 $\left(r_{k}^{-} m_{k}^{-}\right)$, recA1 endA1 gyrA96 thi-1 relA1 & Woodcock et al., 1989 \\
\hline Pseudomonas putida KT2440 & Wild type & Belda et al., 2016 \\
\hline KT2440R & Rif $^{R}$ derivative of $P$. putida KT2440; wild type & Espinosa-Urgel and Ramos, 2004 \\
\hline KT2440RTn7- $\Omega S m 1$ & $\mathrm{Rif}^{\mathrm{R}}, \mathrm{Sm}^{\mathrm{R}}$; extragenic site-specific insertion of mini- $\mathrm{Tn} 7$ & Matilla et al., 2007 \\
\hline KT-PcaY & RifR, $\mathrm{Km}^{\mathrm{R}} ;$ P. putida KT2440R transposon mutant pp2643::mini-tn5- $\Omega \mathrm{Km}$. & Duque et al., 2007 \\
\hline \multicolumn{3}{|l|}{ Plasmids } \\
\hline pET28b(+) & $\mathrm{Km}^{\mathrm{R}}$; Protein expression plasmid & Novagen \\
\hline pBBR1MCS-5 & $\mathrm{Gm}^{\mathrm{R}}$; oriRK2 mobRK2 & Kovach et al., 1995 \\
\hline pET28-PcaY_PP-LBD & $\mathrm{Km}^{\mathrm{R}} ; \mathrm{pET} 28 \mathrm{~b}(+)$ derivative containing DNA fragment encoding PcaY_PP-LBD & This study \\
\hline pMAMV260 & $\mathrm{Gm}^{\mathrm{R}}$; pBBR1MCS-5 derivative containing $p c a Y$ PP gene and its 482 bp upstream region & This study \\
\hline
\end{tabular}

a Km, kanamycin; Rif, rifampin; Gm, gentamicin. 
analysis buffer ( $5 \mathrm{mM}$ PIPES, $5 \mathrm{mM}$ MES, $5 \mathrm{mM}$ Tris- $\mathrm{HCl}$, $\mathrm{pH} 8.0$ ).

\section{Isothermal Titration Calorimetry (ITC)}

Experiments were carried out at $25^{\circ} \mathrm{C}$ using a VP-ITC titration calorimeter (Microcal Inc., Northampton, Massachusetts). Protein at $25 \mu \mathrm{M}$ in analysis buffer was degassed prior to its introduction into the sample cell and titrated with aliquots of $500 \mu \mathrm{M}$ to $3 \mathrm{mM}$ ligand solutions prepared in the same buffer. Obtained data were corrected with the heat changes measured for the injection of ligand into buffer. Data were normalized with the protein concentration (Bradford assay) and fitted with the "One Binding Site" model of the MicroCal version of the ORIGIN 7.0 software (OriginLab Corporation, Northampton, MA, United States), leaving all parameters floating.

\section{Analytical Ultracentrifugation}

Experiments were performed in a Beckman Coulter Optima XL-A analytical ultracentrifuge (Beckman-Coulter, Palo Alto, CA, United States) equipped with UV-visible absorbance detection system, using an An50Ti 8-hole rotor, $12 \mathrm{~mm}$ pathlength charcoal-filled epon double-sector centerpieces. The sedimentation velocity (SV) experiments were carried out at a rotor speed of $40,000 \mathrm{rpm}$ and $10^{\circ} \mathrm{C}$ using $400 \mu \mathrm{l}$ samples in analysis buffer. Protein was at $15-280 \mu \mathrm{M}$ whereas shikimate, protocatechuate and quinate were added, when indicated, at final concentrations of $400 \mu \mathrm{M}$. Analysis buffer with or without ligands was used as reference. Measurements were made at $232 \mathrm{~nm}$ in the absorbance optics mode. A least squares boundary modeling of the SV data was used to calculate sedimentation coefficient distributions with the size-distribution $\mathrm{c}(\mathrm{s})$ method (Schuck, 2000) implemented in the SEDFIT v11.71 software. Buffer density $(\rho=1.00025 \mathrm{~g} / \mathrm{ml})$ and viscosity $(\eta=0.01314$ Poise) at $10^{\circ} \mathrm{C}$ were calculated from the buffer composition using SEDNTERP software (Laue et al., 1992). The partial specific volume $(0.7139 \mathrm{ml} / \mathrm{g})$ and the monomeric molecular weight $(19.2 \mathrm{kDa})$ of PcaY_PP-LBD were also calculated from its amino acid sequence using this software. The multi-speed sedimentation equilibrium experiments were carried out at $10^{\circ} \mathrm{C}$ and 12,000, 14,000, and 18,000 rpm until equilibrium was reached. Sedimentation was followed by measuring absorbance at 235, 240, 280, and $290 \mathrm{~nm}$ depending on the sample concentration. Proteins were measured at six concentrations ranging from 15 to $280 \mu \mathrm{M}$. Generally two wavelengths were used per sample. A monomer-dimer self-association model implemented in SEDPHAT (Vistica et al., 2004) was used for a global fit that accounts for all concentrations, speeds and wavelength.

\section{Construction of Plasmid for Genetic Complementation Assays}

For the construction of the complementing plasmid, the pcaY_PP gene and its promoter region were amplified by PCR using

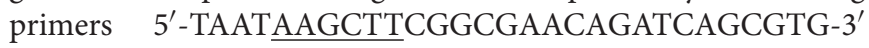
and $5^{\prime}$-TAATGGATCCGCAGAGGTCAGGCAGCGACG-3'. The resulting fragment was digested with HindIII and BamHI and cloned into the same sites in pBBR1MCS-5 to generate pMAMV260. The insert was confirmed by PCR and sequencing, and pMAMV260 was used to transform the $p c a Y \_P P$ defective mutant by electroporation.

\section{Quantitative Capillary Chemotaxis Assays}

Overnight cultures of $P$. putida KT2440 strains were diluted to an $\mathrm{OD}_{660}$ of 0.05 in $\mathrm{M} 9$ minimal supplemented with $10 \mathrm{mM}$ benzoate as carbon source and grown at $30^{\circ} \mathrm{C}$ with orbital shaking $(200 \mathrm{rpm})$. At an $\mathrm{OD}_{660}$ of $\sim 0.4$ (early stationary phase of growth), the cultures were centrifuged at $1,700 \mathrm{~g}$ for $5 \mathrm{~min}$ and the resulting pellet was washed twice with chemotaxis buffer $(50 \mathrm{mM}$ potassium phosphate, $20 \mu \mathrm{M}$ EDTA, $0.05 \%(\mathrm{v} / \mathrm{v})$ glycerol, $\mathrm{pH}$ 7.0). Subsequently, the cells were resuspended in the same buffer, adjusted to an $\mathrm{OD}_{660}$ of 0.15 and $230 \mu \mathrm{l}$ aliquots of this bacterial culture were placed into 96-well plates. For the quantitative assays, one-microliter capillary tubes (Microcaps, Drummond Scientific, Ref. P1424) were heat-sealed at one end and filled with either the chemotaxis buffer (negative control) or chemotaxis buffer containing the chemoeffector. The capillaries were immersed into the bacterial suspension and incubated for $30 \mathrm{~min}$ at room temperature. Capillaries were subsequently removed from the bacterial suspension, rinsed with sterile water and the content expelled into $1 \mathrm{ml}$ of M9 medium. Serial dilutions were plated onto LB medium (containing the appropriate antibiotics) and the number of colony forming units was determined. In all cases, data were corrected with the number of cells that swam into buffer containing capillaries.

\section{Competitive Root Colonization Assays}

Sterilization, germination and inoculation of maize seeds was carried out as described previously, with minor modifications (Matilla et al., 2007). Briefly, sterile seeds were incubated for $30 \mathrm{~h}$ at $30^{\circ} \mathrm{C}$ with a $5 \times 10^{6} \mathrm{CFU} / \mathrm{ml} 1: 1$ mixture of KT2440RTn7$\Omega S m 1$ (wild type) and KT-PcaY (mutant). Thereafter, seeds were rinsed with sterile deionized water and planted in $50 \mathrm{ml}$ Sterilin tubes containing $40 \mathrm{~g}$ of sterile washed silica sand and $10 \%(\mathrm{v} / \mathrm{w})$ plant nutrient solution $\left(5 \mathrm{mM} \mathrm{Ca}\left(\mathrm{NO}_{3}\right)_{2}, 5 \mathrm{mM} \mathrm{KNO}_{3}, 1 \mathrm{mM}\right.$ $\mathrm{MgSO}_{4}, 0.5 \mathrm{mM} \mathrm{KH}_{2} \mathrm{PO}_{4}, \mathrm{pH} 7.0$ ) supplemented with Fe-EDTA $(100 \mu \mathrm{M})$ and micronutrients. Plants were maintained at $24^{\circ} \mathrm{C}$ with a daily light period of $16 \mathrm{~h}$. After 7 days, bacterial cells were recovered from the rhizosphere or from $1 \mathrm{~mm}$ of the main root apex, as described previously (Matilla et al., 2007). Serial dilutions were plated on LB-agar medium supplemented with rifampicin and streptomycin (or kanamycin) to select KT2440RTn7- $\Omega$ Sm 1 or the pcaY_PP mutant strain, respectively.

\section{RESULTS}

\section{PcaY_PP Recognizes Aromatic and Non-aromatic Chemoeffectors Directly}

The initial objective in the study of PcaY_PP was to identify the mode of ligand recognition. Previous research has shown that most individual chemoreceptor LBDs fold into stable 
domains that bind their chemoeffectors with the same affinity as the full-length receptor (Milligan and Koshland, 1993; Matilla and Krell, 2017). To identify the LBD of PcaY_PP (PcaY_PP-LBD), its sequence was analyzed by the DAS transmembrane prediction server (Cserzo et al., 1997) and the DNA fragment encoding the segment between both transmembrane regions, predicted to be four-helix bundle domain, was cloned into an expression vector. The protein was overexpressed in E. coli and purified from the soluble fraction of its cell lysate. Subsequently, the purified protein was submitted to Isothermal Titration Calorimetry (ITC) binding experiments (Figure 1). The derived dissociation constants are reported in Figure 2 whereas the corresponding changes in enthalpy and Gibbs free energy are reported in Supplementary Table 2.

Prior to all protein titrations, ligands were injected into buffer to determine the corresponding dilution heats (upper traces in Figure 1). The titration of the protein with benzoate resulted in exothermic heats that diminished as protein saturation advanced and a $K_{\mathrm{D}}$ of $90 \pm 8 \mu \mathrm{M}$ was obtained (Figure 1A). No binding heats were observed for the linear $\mathrm{C} 6$ carboxylate adipate (Figure 1A), illustrating the importance of the $\mathrm{C} 6$ ring in the molecular recognition. In all experiments where an absence of binding was noted, the protein-ligand mixture was titrated with benzoate to verify protein integrity. In addition, when an absence of binding was noted, the experiment was repeated at a different analysis temperature to verify whether potential endothermic and exothermic contributions may cancel out each other at a given analysis temperature. Subsequent studies were aimed at determining the effect of different benzoate substitutions on binding. To this end, the three isomers of hydroxy-, amino-, methyl-, chloro-, and nitro- benzoates were analyzed (Figure 2). The 4-hydroxy substitution of benzoate resulted in an around 12 -fold increase in affinity $\left(K_{\mathrm{D}}=7.2 \mu \mathrm{M}\right)$ as compared to benzoate and was by far the tightest binding monosubstituted benzoate derivative (Figures 1B, 2). In contrast, 4-hydroxybenzaldehyde was devoid of binding (Figure 1B) demonstrating the essential role of the carboxyl group for binding. Of the remaining 14 singly substituted benzoates tested, PcaY_PP bound 10 of them with $K_{\mathrm{D}}$ values between $24 \mu \mathrm{M}$ for 2-hydroxybenzoate (salicylate) and $138 \mu \mathrm{M}$ for 3aminobenzoate (Figure 2). Only two 2-substituted derivatives showed binding whereas all five 4 -substituted benzoates bound (Figures 1B,C, 2).

Additionally, we analyzed the three doubly substituted benzoates, protocatechuate, vanillate, and vanillin. Interestingly, the former two compounds had a relatively high affinity, with $K_{\mathrm{D}}$ values of 6.4 and $11 \mu \mathrm{M}$, respectively, that were comparable to that of 4-hydroxybenzoate $\left(K_{\mathrm{D}}=7.2 \mu \mathrm{M}\right)$ (Figures 1D, 2), the tightest binding mono-substituted benzoate. The absence of binding for vanillin underlines the necessity of a carboxyl group for recognition. Finally, the non-aromatic compounds shikimate, quinate and 3-dehydroshikimate were also tested. Surprisingly, from all compounds tested, these non-aromatics showed the highest affinity (Figures 1D, 2). Consequently, PcaY_PP recognizes different aromatic and non-aromatic chemoeffectors directly. However, the receptor recognizes the non-aromatic chemoeffectors with higher affinity. Importantly, of the 24 ligands tested, $75 \%$ of them are natural compounds (marked with an asterisk in Figure 2 and Supplementary Table 3). In addition, recognition of all ligands was driven by favorable enthalpy changes that were counterbalanced by unfavorable entropy changes (Supplementary Table 2).

Aromatic acid responsive chemoreceptors MCP2201 and MCP2983 of C. testosteroni are stimulated by the binding of different TCA cycle intermediates ( $\mathrm{Ni}$ et al., 2013a, 2015). The titration of PcaY_PP-LBD with cis-aconitate, succinate and malate did not show any binding (data not shown).

\section{Ligand Binding Stabilizes the PcaY_PP-LBD Dimer}

Four-helix bundle domains are ubiquitous sensor domains in bacterial signal transduction systems (Ulrich and Zhulin, 2005). In order to determine the effect of ligand binding on the oligomeric state of PcaY_PP-LBD, we carried out analytical ultracentrifugation studies (AUC). In initial sedimentation velocity assays sedimentation coefficients of ligand-free PcaY_PP-LBD were determined at three different concentrations (Figure 3). Data showed a single species (Figure 3) with a sedimentation coefficient that is slightly dependent on the protein concentration. At the intermediate concentration of $25 \mu \mathrm{M}$, the corrected standard $s_{20, w}$ is $2.6 \mathrm{~S}$, with a frictional ratio of 1.4. This corresponds to a virtual intermediate species with a molecular weight of $31 \mathrm{kDa}$, which is between the monomeric and the dimeric species. This virtual species corresponds to rapidly interchanging monomers and dimers and the dimerization-dissociation kinetics is faster than the timescale of the AUC experiment. Similar virtual species for recombinant LBDs have been observed previously (Martin-Mora et al., 2016).

When this analysis was repeated in the presence of saturating concentrations of quinate and shikimate, a single species was observed, but its sedimentation coefficient had shifted to $s_{20, w}=3.1 \mathrm{~S}$, with a frictional ratio of 1.3 (slightly elongated). The molecular weight calculated for this species from the sedimentation coefficient corresponds to $38.0 \mathrm{kDa}$, which is very close to the sequence derived molecular weight of the PcaY_PP-LBD dimer of $38.4 \mathrm{kDa}$. It can therefore be concluded that the binding of ligands stabilizes the PcaY_PP-LBD dimer.

The concentration dependence of the sedimentation coefficient of ligand-free PcaY_PP-LBD and the fact that the single peak corresponds to a virtual intermediate species suggest that the protein is present in a monomer-dimer equilibrium. To characterize this equilibrium we conducted a multi-speed and multi-wavelength sedimentation equilibrium experiments covering the protein concentration range of 15 to $280 \mu \mathrm{M}$ (Figure 4). When data were globally fitted to a monomerdimer model a $K_{\mathrm{d}}=57.5 \mu \mathrm{M}$ was obtained for the protein auto-association (Figure 4). This value, combined with the observation that binding in ITC was only observed at higher protein concentrations (above $5 \mu \mathrm{M}$ ), indicates that only dimeric PcaY_PP-LBD is able to bind ligands which in turn causes dimer stabilization. 
A

Time ( $\mathrm{min})$

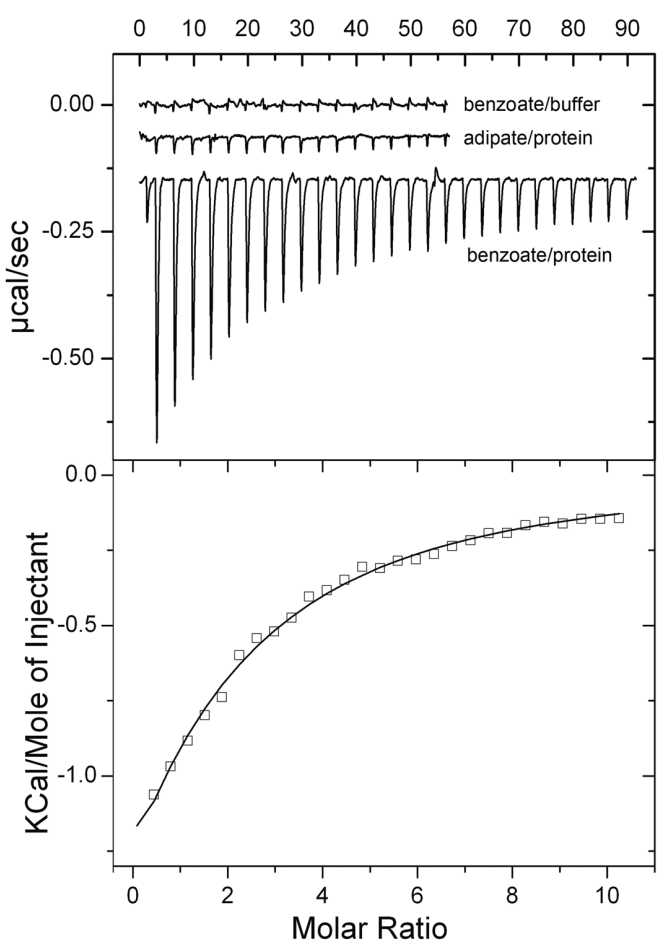

C

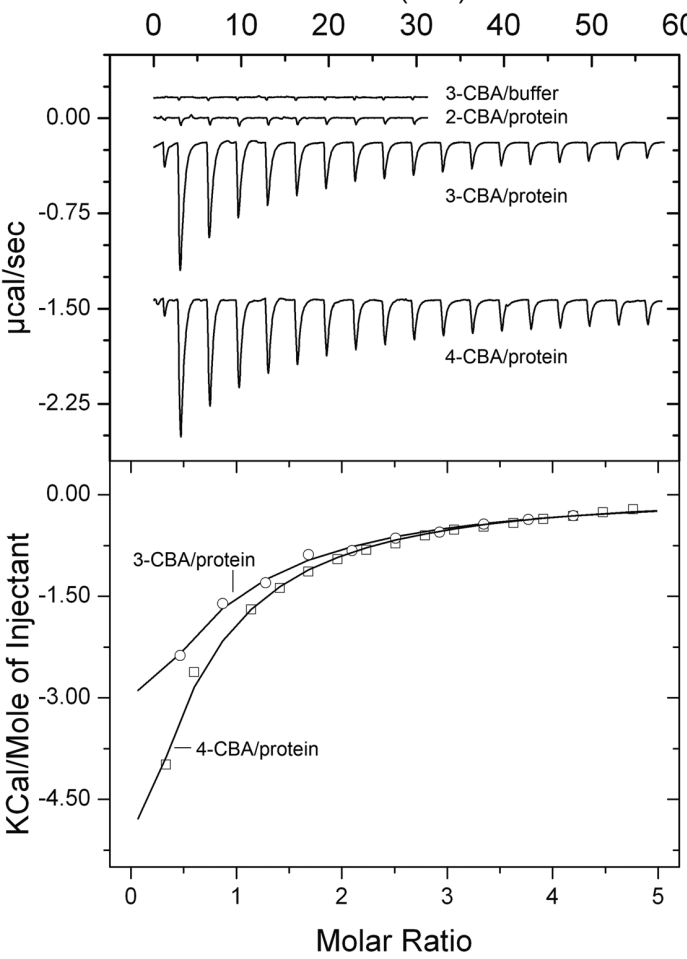

B

Time (min)

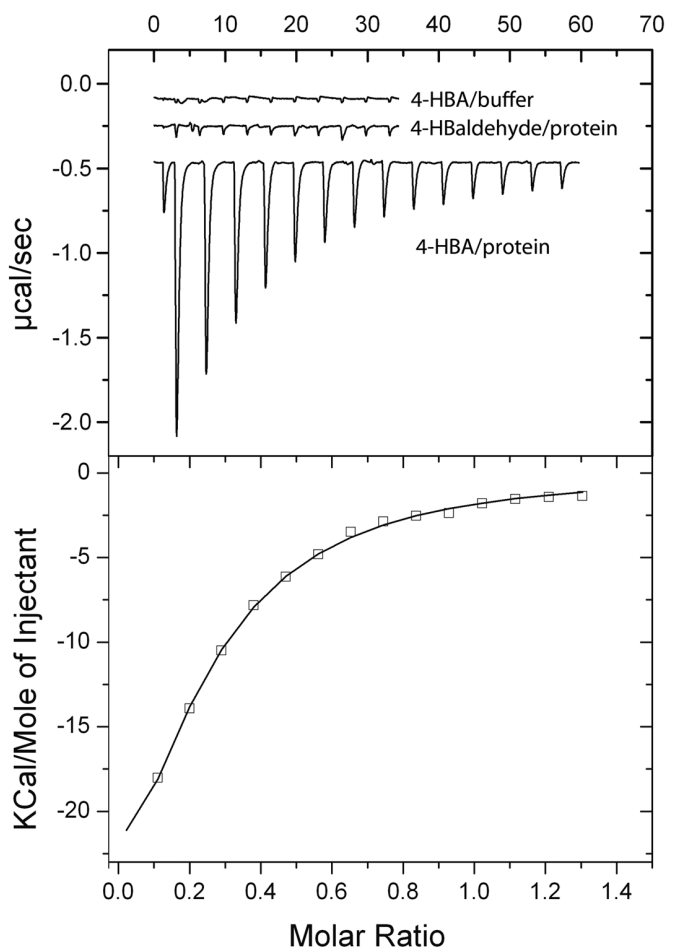

Time (min)

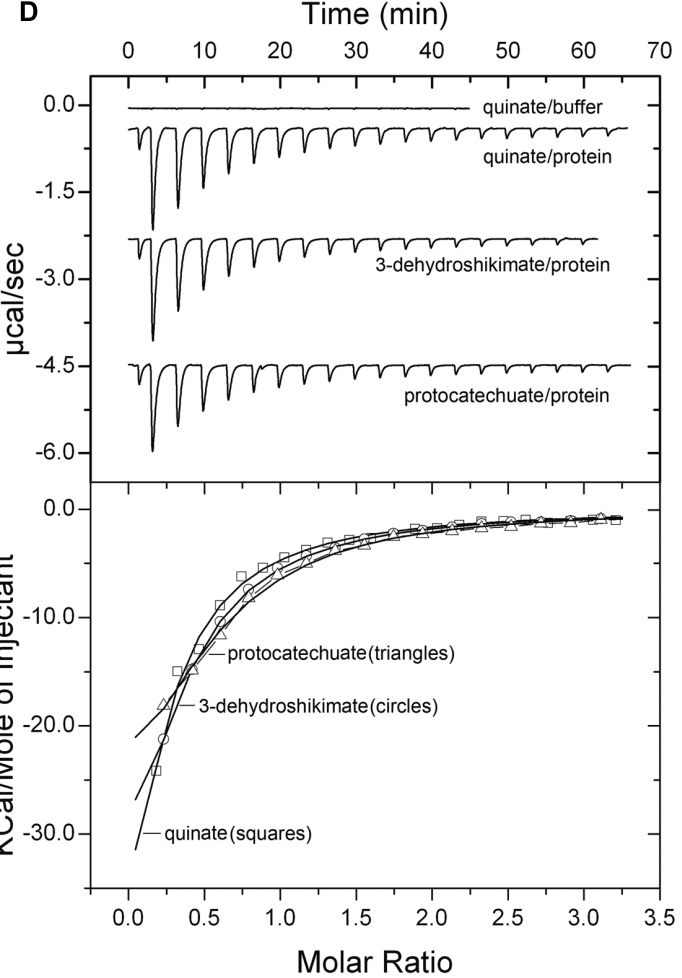

FIGURE 1 | Microcalorimetric studies of the binding of different ligands to purified PcaY_PP-LBD. Upper panels: raw titration data; the upper traces correspond to the injection of ligand into buffer (control). Lower panels: Integrated, concentration-normalized and dilution heat corrected peak areas of the raw data. The line is the best fit to the "One binding site model" of the MicroCal version of ORIGIN. Protein concentration was of $25 \mu \mathrm{M}$, injection volumes were between 4.8 and $9.6 \mu \mathrm{I}$ and ligand concentrations between 0.5 and $2 \mathrm{mM}$. Titration with benzoate and adipate (A); 4-hydroxybenzoate and 4-hydroxybenzaldehyde (B); 2-chlorobenzoate,

3-chlorobenzoate and 4-chlorobenzoate (C); quinate, 3-dehydroshikimate and protocatechuate (D). 


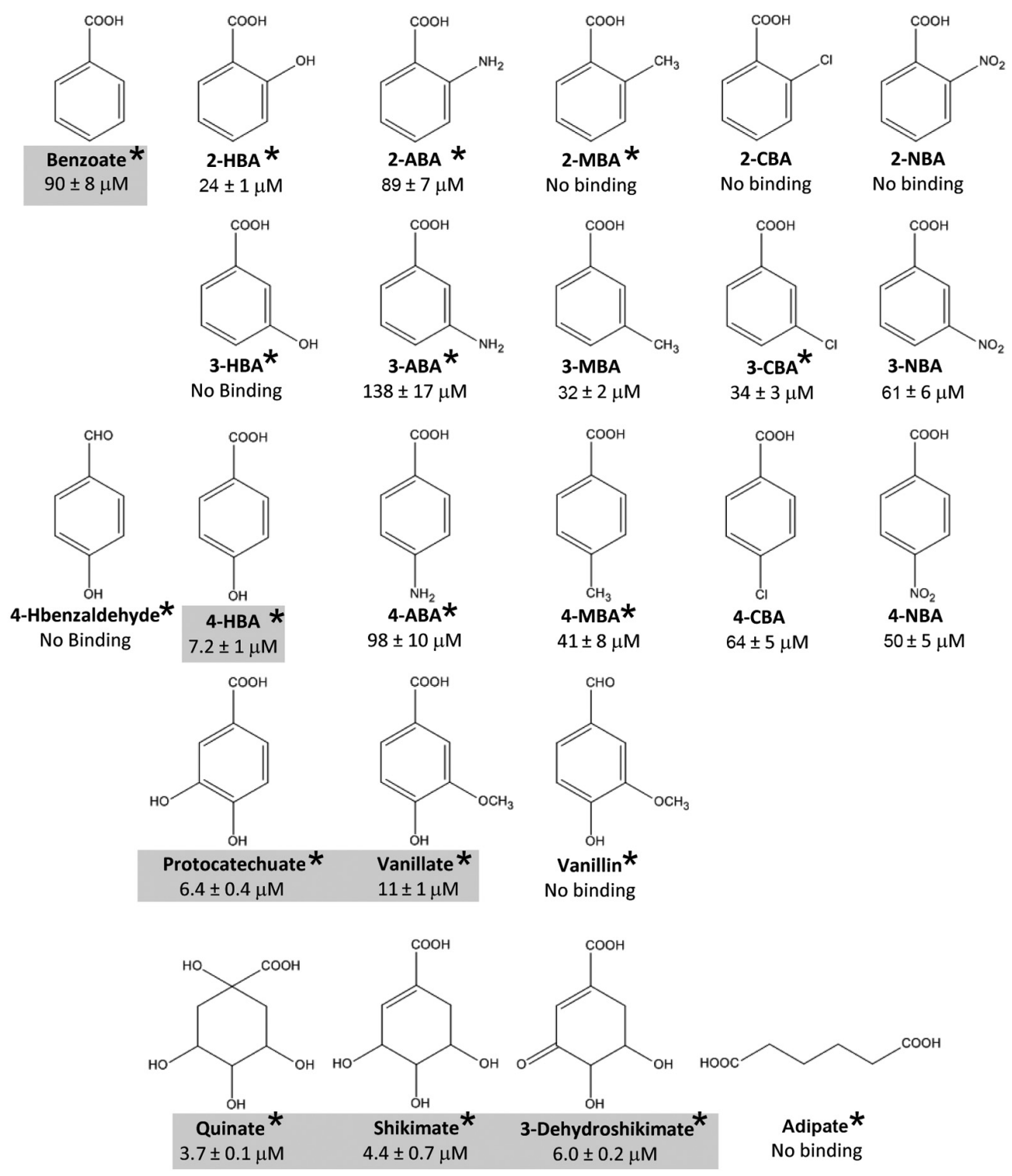

FIGURE 2 | Summary of results from microcalorimetric titrations of PcaY_PP-LBD with different ligands. Shown are structures and the corresponding $K_{D}$ values as determined by ITC. The gray shading indicates the capacity to support growth of KT2440 as sole carbon source. Data are means and standard deviations from three experiments. Asterisks indicate that it is a natural compound according to the Zinc database (Irwin et al., 2012). For further information on these compounds see Supplementary Table 3.

\section{Different Magnitude of Chemotactic Response to PcaY_PP Ligands}

Subsequently, we investigated the chemotactic responses of P. putida KT2440 to PcaY_PP ligands. To this end, we selected seven compounds that differ in structure and affinity and have conducted quantitative capillary chemotaxis assays (Figure 5). Among these compounds was a single compound, 2-chlorobenzoate, that did not bind to PcaY_PP-LBD. As expected this compound did not induce a chemotactic response
(Figure 5). Relevant chemotaxis was observed for the remaining six compounds at a concentration range between $100 \mu \mathrm{M}$ to $100 \mathrm{mM}$, with optimal responses at $10 \mathrm{mM}$. Interestingly, significant differences were observed in the magnitude of response. Whereas shikimate and quinate produced very strong responses, those of protocatechuate, 2-hydroxybenxoate (salicylate), benzoate and 4-aminobenzoate were much weaker (Figure 5). The magnitude of chemotaxis correlated with the $K_{\mathrm{D}}$ values as determined above (Supplementary Figure 2), indicating 


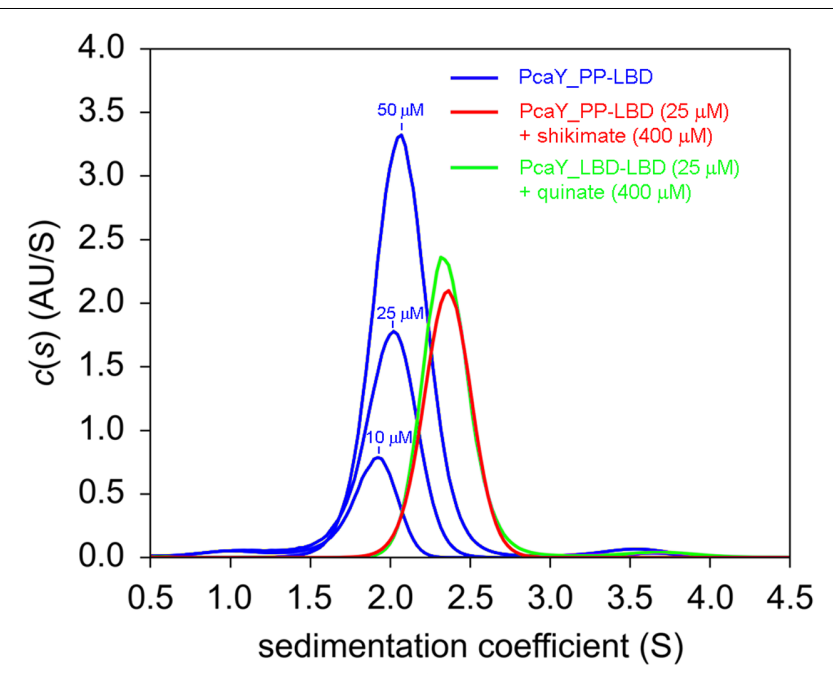

FIGURE 3 | Sedimentation velocity analytical ultracentrifugation studies of PcaY_PP-LBD. Sedimentation coefficient profiles obtained for protein in the absence and presence of ligands.

that the affinity is a key parameter that defines the magnitude of chemotactic responses.

To assess the contribution of PcaY_PP to chemotaxis, we characterized a transposon mutant defective in the $p c a Y \_P P$ gene. Initial control experiments involved the assessment of chemotaxis of the wild-type and mutant strain to casamino acids. As shown in Supplementary Figure 3, the inactivation of $p c a Y \_P P$ did not alter amino acid chemotaxis, indicating that the inactivation of this gene did not have any undesired secondary effects. Figure $\mathbf{6 A}$ illustrates that the inactivation of the PcaY_PP gene abolished chemotaxis to the four structurally different chemoattractants studied. To confirm the association between the transposon mutation and the chemotactic phenotype, genetic complementation studies were carried out. For these assays, the $p c a Y \_P P$ gene and its promoter region were cloned into plasmid pBBR1MCS-5 and the resulting pMAMV260 was introduced into the $p c a Y \_P P$ mutant strain. For control purposes, the empty plasmid pBBR1MCS-5 was also introduced into the wt and mutant strains. The resulting strains were submitted to chemotaxis assays to $10 \mathrm{mM}$ solutions of the four selected compounds. Data shown in Figure 6B illustrate that the chemotaxis of the complemented mutant was similar to that of the wt strain bearing empty plasmid pBBR1MCS-5. Taken together, data indicate that PcaY_PP is the physiologically relevant receptor for these compounds in P. putida KT2440.

\section{Inactivation of pcaY_PP Does Not Affect Root Colonization}

For root-associated bacteria, chemotaxis to plant root exudates is an essential prerequisite for efficient root colonization (Scharf et al., 2016). We have shown recently that the mutation of $m c p G$ and $m c p U$, encoding chemoreceptors for gamma-aminobutyrate and polyamines, respectively, reduced plant root colonization capabilities by P. putida KT2440 (Reyes-Darias et al., 2015a; Corral-Lugo et al., 2016). The composition of root exudates is very complex and strong attractants such as quinate, shikimate, salicylate, or benzoate were identified in plant root exudates (Khorassani et al., 2011; Tawaraya et al., 2014; Bowsher et al., 2016). To investigate the implications of PcaY_PP in the efficient colonization of the rhizosphere, we conducted competitive root colonization studies. In these assays, maize roots were inoculated with 1:1 mixtures of the wt and $p c a Y \_P P$ mutant strain. After 7 days, bacteria were recovered from the rhizosphere and the root tip for quantification. As shown in Supplementary Figure 4, the ratios of recovered wt to mutant cells were approximately $1: 1$,
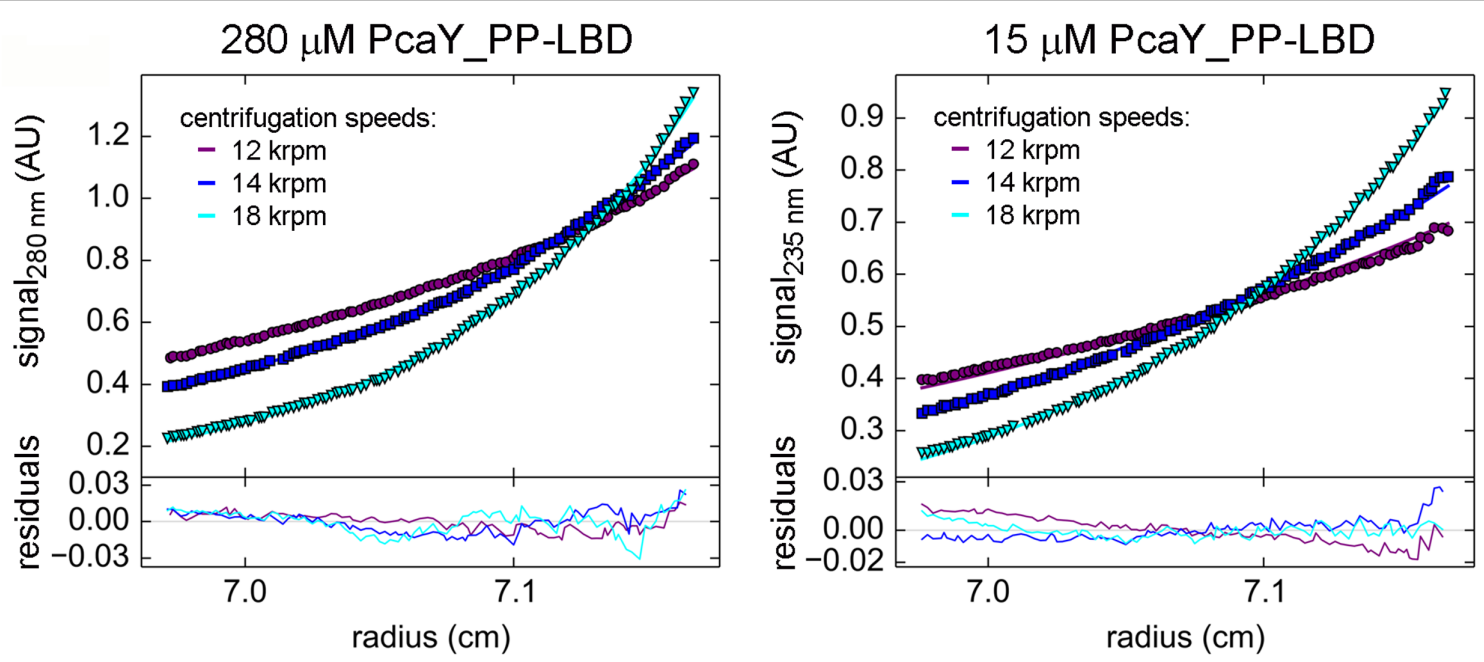

FIGURE 4 | Sedimentation equilibrium analytical ultracentrifugation studies of PcaY_PP-LBD. Absorbance profiles of two different concentrations of ligand-free PcaY_PP-LBD at three different speeds. The experimental values are represented by filled symbols while the line represents the best global fit to these values for the six concentrations, three speeds, and two wavelengths (30 curves) used in this experiment. 


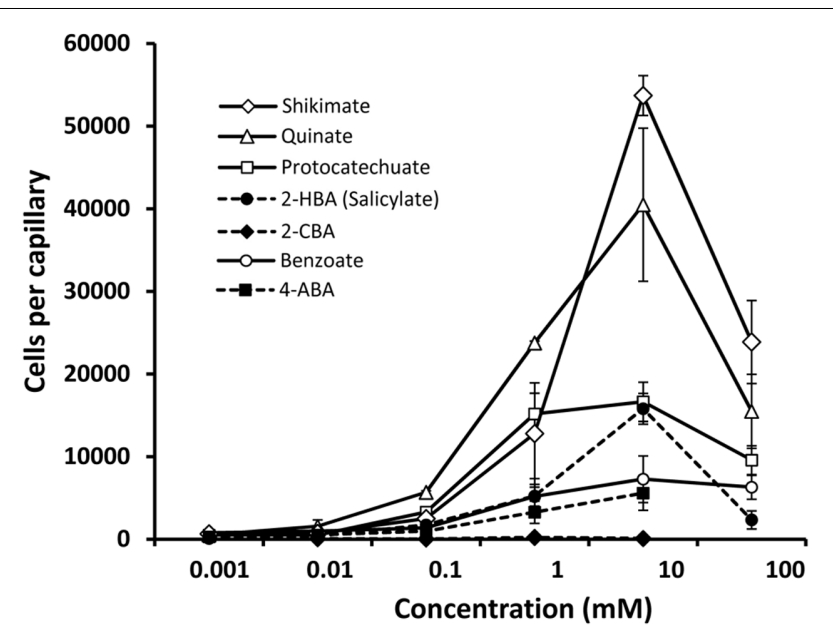

FIGURE 5 | Quantitative capillary chemotaxis assays of Pseudomonas putida KT2440 toward different compounds. Data are means and standard deviations from three biological replicates conducted in triplicate. Data were corrected with the number of cells that swam into buffer containing capillaries $(1443 \pm 370)$

indicating that $p c a Y \_P P$ inactivation did not alter competitive plant root colonization.

\section{Only Seven of the Ligands Identified Support Bacterial Growth As Sole Carbon Source}

Binding studies have resulted in the identification of 17 ligands recognized by PcaY_PP. To assess which of these compounds are able to sustain growth of $P$. putida KT2440 as sole carbon source, we conducted growth experiments in M9 minimal medium supplemented with each of the PcaY_PP ligands. Only seven ligands supported bacterial growth, which were benzoate, 4-hydroxybenzoate, protocatechuate, vanillate, quinate, shikimate, and dehydroshikimate (gray shading in Figure 2). Of the 15 singly substituted benzoate derivatives, only a single compound, 4-hydroxybenzoate, supported growth. Interestingly, the affinity of this compound was significantly higher than those of all other singly substituted benzoates (Figure 2). As exemplified in Supplementary Figure 5 for quinate and benzoate, there were significant differences in the growth kinetics. From these growth experiments, we have derived bacterial yields as well as generation- and lag times. In subsequent analyses, we determined whether the chemoeffector affinity as determined by ITC correlates with any of these parameters. As shown in Supplementary Figure 6 no correlation was observed between the affinity and the yield or generation time. In contrast, differences observed in the lag phase of bacterial cultures correlated with the chemoeffector $K_{\mathrm{D}}$ for the chemoreceptor (Figure 7). In a second analysis, we introduced the $K_{\mathrm{D}}$ values of the 10 PcaY_PP ligands that did not support into the graph of Figure 7 . These compounds grouped together in the weaker affinity range. These data were submitted to a $\chi^{2}$ test for a two-category statistical analysis defining the categories as ligands that can or cannot be

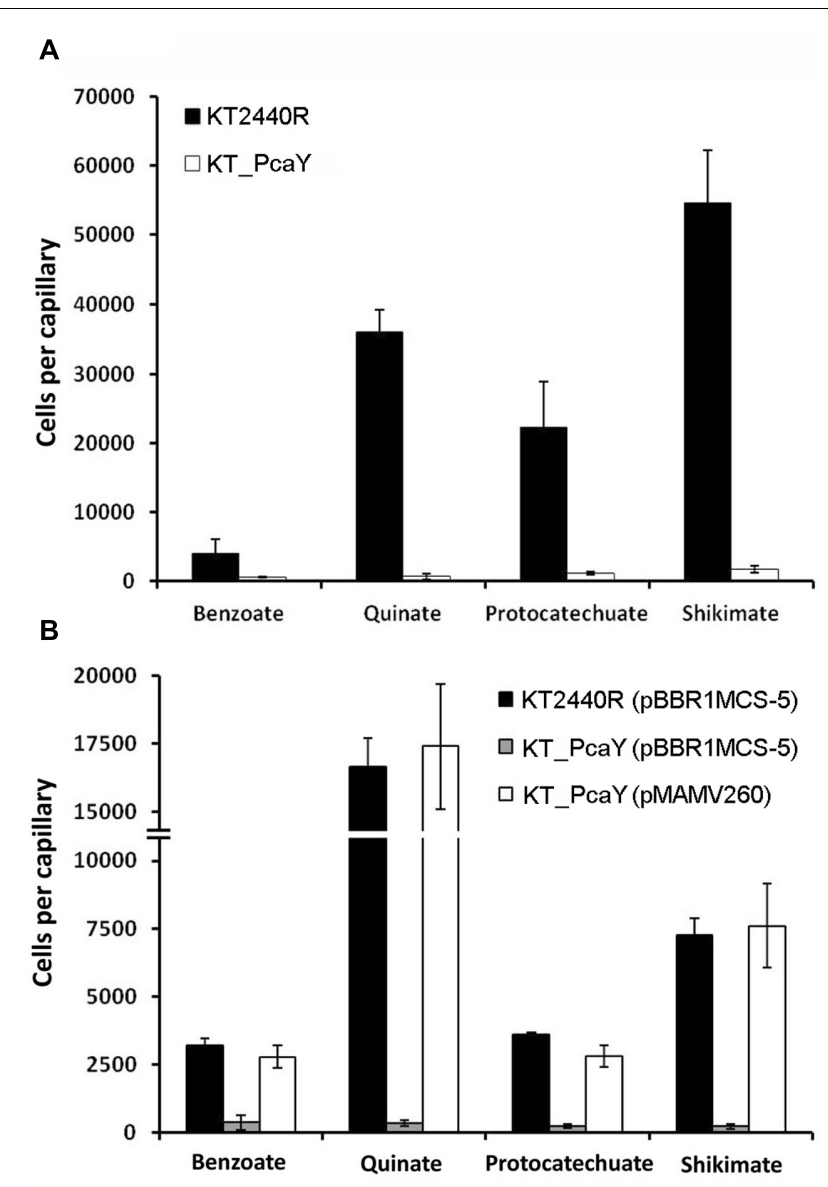

FIGURE 6 | Quantitative capillary chemotaxis assays of $P$. putida KT2440R, its mutant defective in $p c a Y \_P P$ and the complemented mutant strains to different PcaY_PP chemoeffectors. (A) Chemotaxis of the wt and its pcaY_PP mutant to different chemoeffectors. (B) Complementation assays of the pcaY_PP mutant by the in trans expression of pcaY_PP using the pBBRMCS-5-based vector, pMAMV260. As controls, chemotaxis of the wt and mutant strain harboring empty plasmid pBBRMCS-5 were analyzed. The chemoeffector concentration was $10 \mathrm{mM}$. Data were corrected with the number of cells that swam into buffer containing capillaries (920 \pm 105 for $\mathbf{A}$; $148 \pm 34$ for B). Data are the means and standard deviations from three biological replicates conducted in triplicate.

used as C-sources and compounds having high $\left(K_{\mathrm{D}}<20 \mu \mathrm{M}\right)$ and low $\left(K_{\mathrm{D}}>20 \mu \mathrm{M}\right)$ affinity. A $\chi^{2}$ of 3500 and a corresponding $P$-value of 0.03 was obtained indicating a correlation between the chemoeffector affinity for its chemoreceptor and the capacity to sustain growth as sole C-source. Taken together, there are several pieces of evidence indicating a relationship between the metabolic value of compounds and their recognition by the PcaY_PP chemoreceptor.

\section{DISCUSSION}

In the model organism of this study, P. putida KT2440, eight chemoreceptors have been functionally characterized (Supplementary Table 1). These receptors were shown to be 


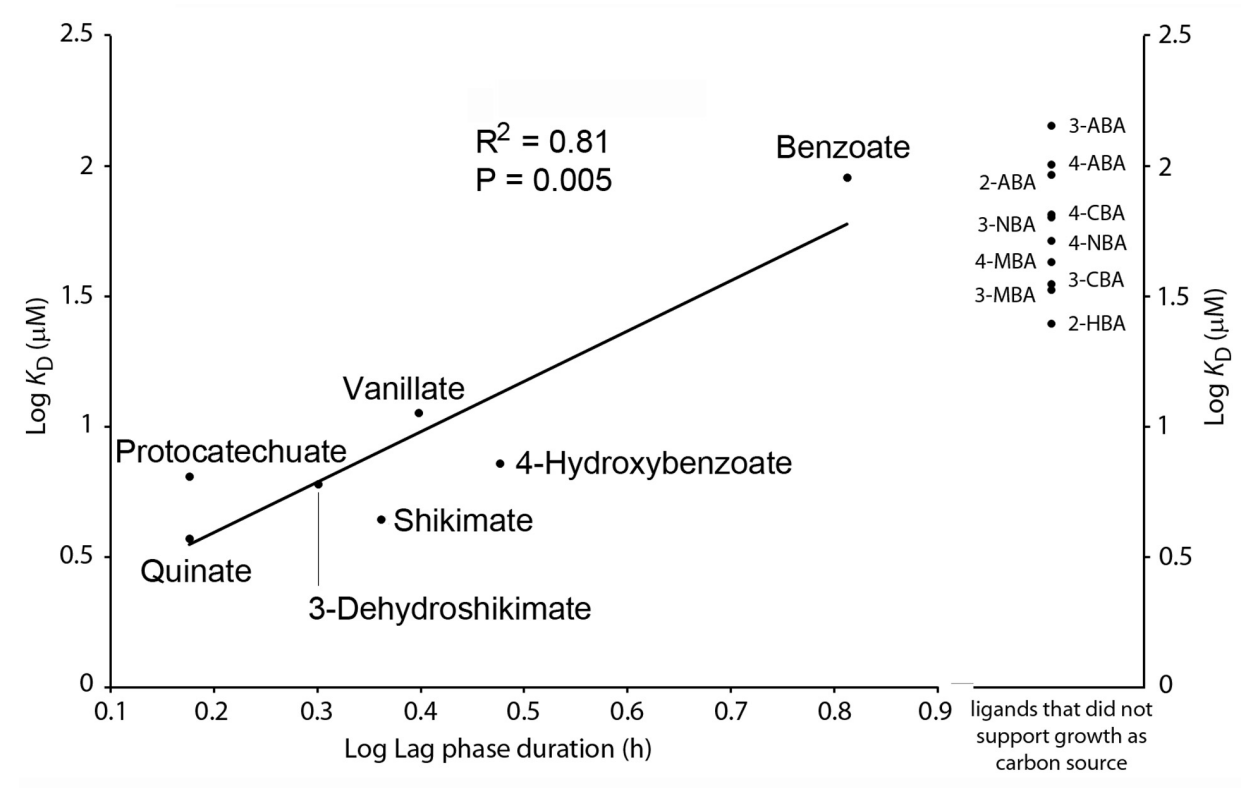

FIGURE 7 | Relationship between binding affinity of chemoeffectors to PcaY_PP-LBD and the capacity of these compounds to support bacterial growth as sole C-source. Left: Plot of the dissociation constants as determined by ITC (see Figure 2) and the duration of the lag time during growth on minimal medium containing the compound indicated a sole carbon source. Data were fitted by linear regression. Right: Dissociation constants obtained for PcaY_PP ligands that did not support growth as sole carbon source.

activated by the direct binding of 34 different chemoeffectors, which are all of metabolic value since they support growth as Cand/or N-sources. The PcaY_PP receptor seems to be different since only some of the identified ligands supported growth as sole C-source (Figures 2, 7). As illustrated in Figure 5, ligands of both classes cause chemoattraction.

Chemotactic signaling is decoupled from uptake and metabolism of compounds. We report here several pieces of evidence suggesting that the metabolic capacity of the chemoeffectors is an important parameter in the recognition of ligands by PcaY_PP. Firstly, there was a correlation between the duration of the growth lag time and the dissociation constant (Figure 7). Secondly, the $K_{\mathrm{D}}$ values of non-metabolizable ligands were significantly higher than those of metabolizable ligands (Figures 2, 7). Thirdly, of the 15 singly substituted benzoate derivatives, there was only one, 4-hydroxybenzoate, that served for growth. The $K_{\mathrm{D}}$ value of this compound $(7.2 \pm 1 \mu \mathrm{M})$ was significantly lower than that of all other singly substituted benzoates, which had an average $K_{\mathrm{D}}$ of $63 \pm 35 \mu \mathrm{M}$. Striking parallels exist to a study that evaluated the relationship between chemotaxis and metabolism to amino acids, which are among the most valuable nutrient sources for bacteria (Yang et al., 2015). In E. coli there was a nearly perfect overlap between chemoattractive and catabolized amino acids. Seven amino acids were identified as specific chemoattractants, of which six were rapidly consumed, whereas only one amino acid that served for growth was not an attractant. In addition, a second correlation was observed between the order of amino acid utilization and their efficiency as chemoattractants. The authors conclude that the chemotaxis system of $E$. coli has evolved to specifically locate sources of most nutritionally valuable amino acids (Yang et al., 2015). In marked contrast no such correlations were reported for Bacillus subtilis and the authors speculated that $B$. subtilis rather uses gradients of amino acids as general cues to locate sources of nutrients such as plant roots or to initiate symbiosis or pathogenesis. Our data indicate a similar chemotaxis-metabolism relationship as observed for $E$. coli. Data reported furthermore indicate that the modulation of the binding affinity at the chemoreceptor is a mechanism by which the metabolic potential of compounds reflects on the magnitude of chemotaxis.

The dissociation constants of different ligands to the purified LBDs of all characterized P. putida KT2440 chemoreceptors are shown in Supplementary Table 4. All constants were determined using isothermal titration calorimetry using very similar experimental conditions. Of these receptors only one binds specifically one compound, namely the gammaaminobutyrate (GABA) specific McpG (Reyes-Darias et al., 2015a), whereas all other receptors recognize multiple ligands. Among all receptors, GABA binding at McpG-LBD showed the highest affinity $\left(K_{\mathrm{D}}=0.17 \mu \mathrm{M}\right)$, which is consistent with the notion that ligand recognition is a trade-off between affinity and specificity. The dissociation constants of the PcaY_PPLBD ligands, as determined here, are in the same range as the constants for the remaining characterized P. putida KT2440 receptors (Supplementary Table 4). The data reported also reveal the minimal ligand requirements for binding, which consist in a 6-membered carbon ring and a carboxylic acid group. For the recognition of aromatic compounds, the substitution at the C2-position was an essential criteria (only hydroxyl and amino substituents bound), whereas the nature of substitutions at the 


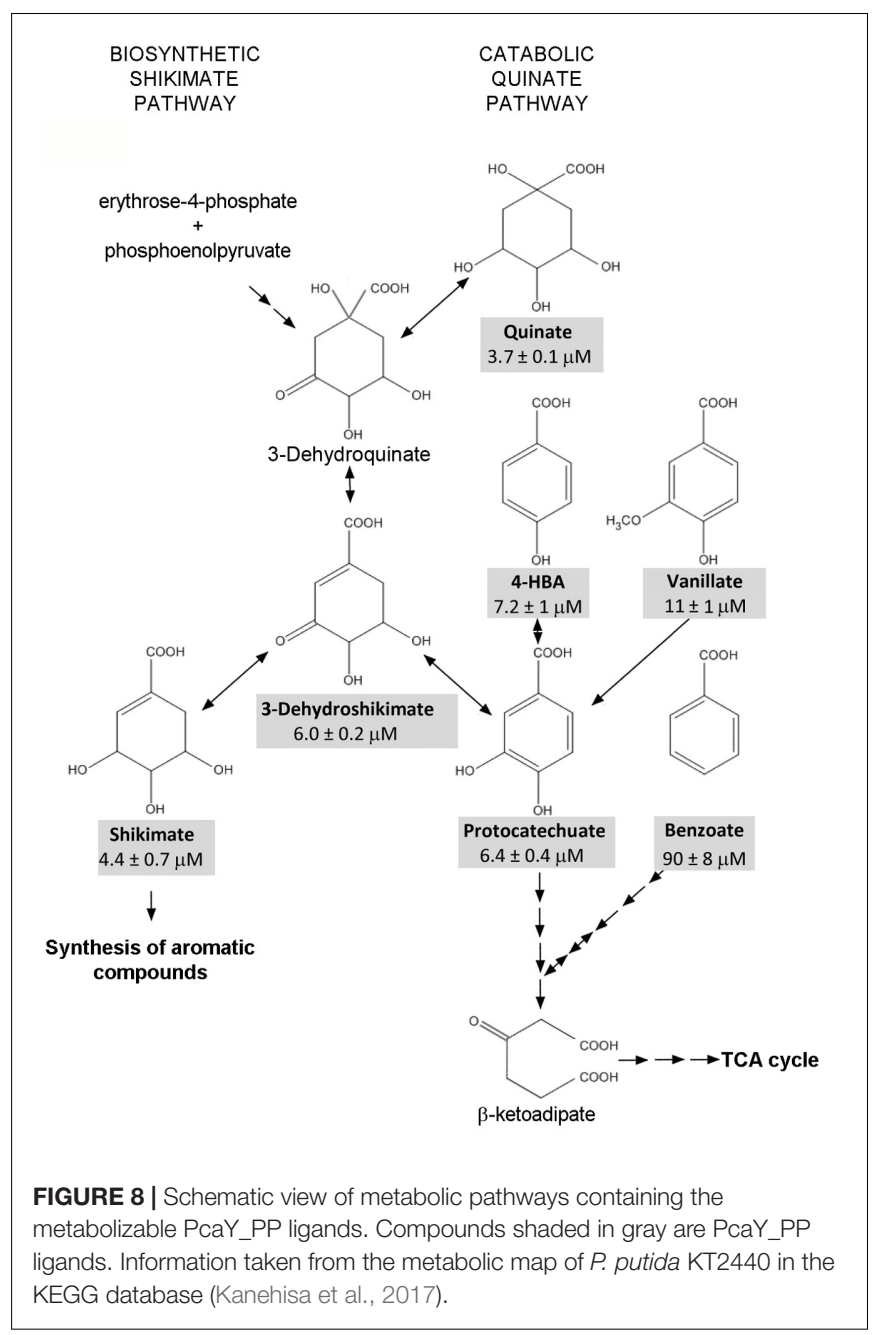

C3- and C4-positions were less critical (Figure 2). In this context interesting parallels exist to the broad range aromatics sensing TodS histidine kinase, where C2-substituents determined largely the magnitude of regulatory response (Busch et al., 2007).

PcaY (Luu et al., 2015) and PcaY_PP share a highly similar ligand spectrum and in both cases a significant number of chemoeffectors were found to lack metabolic value. The questions that remained unanswered in Luu et al. (2015) were, firstly, whether the chemoeffectors identified or metabolic derivatives thereof, like in the case of the $C$. testosteroni aromatic acid receptors ( $\mathrm{Ni}$ et al., 2013a, 2015), activated the receptor and, secondly, whether this activation occurred via direct binding or an alternative indirect mechanism. We show here that receptor activation is achieved through the direct binding of chemoeffectors to the LBD of the receptor. Another question concerns the potential physiological benefit of chemotaxis to compounds that are of no apparent value. So far no answer can be given to this question, but data on PcaY and PcaY_PP show clear parallels to the recognition of aromatic compounds by other bacterial sensor proteins. Thus, due to the action of the toluene dioxygenase (TOD) pathway, $P$. putida DOT-T1E can metabolize benzene, toluene and ethylbenzene and use these compounds as sole carbon sources. However, the McpT chemoreceptor of this strain mediates chemotaxis, apart from these three TOD substrates, to an extensive array of structurally related but non-metabolizable mono- and biaromatic compounds (Lacal et al., 2011). Very similar observations have been made in the study of the TodS sensor kinase that controls the expression of the TOD pathway operon. Apart from the three substrates, TodS recognizes many different non-metabolizable aromatic compounds and induces pathway expression in their response (Lacal et al., 2006; Busch et al., 2007). Taken together, it can be speculated that these chemotactic and transcriptional responses to compounds that lack apparent metabolic value may be related to the molecular complexity of evolving molecular recognition mechanism that are specific for certain aromatic compounds. As a consequence, some sensor proteins may have evolved to recognize many different ligands of which only some are of metabolic value. It is plausible that of compounds without any apparent metabolic value may represent environmental cues and their detection may be beneficial to the bacterium as proposed by (Yang et al., 2015).

We show here that the chemoeffector affinity for PcaY_PP determines the magnitude of the chemotactic response (Supplementary Figure 2), which is a correlation that has already observed for other broad range chemoreceptors (ReyesDarias et al., 2015b) but that does not apply to all chemoreceptors (Lacal et al., 2010). The tightest binding ligand was quinate, followed by shikimate, 3 -dehydroshikimate and protocatechuate. These compounds are located at the intersection of two metabolic pathways, namely the catabolic quinate pathway that feeds into the $\beta$-ketoadipate pathway and subsequently into the TCA cycle, and the shikimate pathway for the biosynthesis of most aromatic compounds (Figure 8). In fact, 3-dehydroshikimate is an intermediate of both pathways. Chemotaxis to these ligands is therefore of double benefit since it permits access to C- and energy sources as well as to compounds that are required for the biosynthesis of aromatic compounds. Parallels exist to other chemoreceptors where receptor ligands are equally at the cross-roads of catabolic and biosynthetic pathways. One such example is the $\mathrm{McpH}$ chemoreceptor that is responsible for chemotaxis to adenine and guanine (Fernandez et al., 2016). Both compounds are at the beginning of the purine degradation pathway for their conversion into urea which can be used as sole $\mathrm{N}$-source. Both bases, however, also feed into the biosynthetic pathway of purine nucleotides. In the particular case of quinate, this cyclohexanecarboxylic acid is abundantly present in many different plants (Bentley, 1990; Lehmann et al., 2016) and was shown to accumulate to concentrations above $50 \%$ of the plant dry weight (Bhatia et al., 2015), suggesting that it may serve as carbon storage compound. It is therefore not astonishing that a plant saprophyte like $P$. putida KT2440 has a high-affinity chemoreceptor for quinate.

Several studies show that P. putida KT2440 is able to efficiently colonize the rhizosphere in non-sterilized soils, i.e., in the presence of naturally occurring soil bacteria (Molina et al., 2000; Matilla et al., 2010; Neal et al., 2012; PizarroTobias et al., 2015). In addition, competition assays with other P. putida, P. fluorescens, and P. chlororaphis strains show 
that KT2440 is equally efficient in rhizosphere colonization (Molina et al., 2000). Chemotaxis was found to be essential for efficient root colonization (Scharf et al., 2016). Although the PcaY_PP chemoreceptor appears to be dispensable for efficient root colonization (Supplementary Figure 4), other KT2440 chemoreceptors like the GABA specific McpG (Reyes-Darias et al., 2015a) and the polyamine specific McpU (Corral-Lugo et al., 2016) were found to be necessary for optimal root colonization.

Similar to KT2440, C. testosteroni CNB-1 shows chemoattraction to aromatic acids like benzoate, 3-hydroxybenzoate, 4-hydroxybenzoate, protocatechuate, vanillin, or vanillate (Ni et al., 2013a). All of these compounds support the growth of the bacterium (Ni et al., 2013b) and further studies showed that the chemoreceptors MCP2201, MCP2901, and MCP2983 mediate taxis to these compounds (Ni et al., 2013a, 2015; Huang et al., 2016). These receptors possess a four-helix bundle LBD but employ different mechanisms. Surprisingly, the recombinant LBDs of receptors MCP2201 and MCP2983 did not bind any of the aromatic acids. Instead, MCP2201-LBD bound a series of TCA cycle intermediates ( $\mathrm{Ni}$ et al., 2013a), whereas MCP2983 recognized cis-aconitate ( $\mathrm{Ni}$ et al., 2015). These compounds are generated by the metabolism of aromatic acids and it was proposed that MCP2201- and MCP2983-mediated chemotaxis to aromatic acids is caused by the recognition of the resulting metabolites ( $\mathrm{Ni}$ et al., 2013a). This hypothesis was supported by the fact that the observed chemotaxis was metabolism dependent ( $\mathrm{Ni}$ et al., 2013a). In marked contrast, the mechanism of MCP2901 is based on the direct binding of aromatic acids to the periplasmic LBD (Huang et al., 2016); although affinities were relatively weak $\left(K_{\mathrm{D}}\right.$ values typically of several hundreds of $\mu \mathrm{M}$ ) and thus inferior to the corresponding values of PcaY_PP. Taken together, there are thus two fundamentally different mechanism of chemotaxis to aromatic acids. One mechanism (MCP2201 and MCP2983) is based on the recognition of TCA cycle intermediates that are generated by the aromatic acid metabolism, whereas the mechanism of MCP2901 and PcaY_PP relies on the direct recognition of aromatic acids by periplasmic LBDs.

The Tar receptor is the primary model in the study of chemoreceptors. Classical biochemical studies showed that the recombinant Tar-LBD is present in a monomer-dimer equilibrium and the self-association constant was estimated to be in between 0.5 and $5 \mu \mathrm{M}$. Aspartate binding was shown to increase the dimer formation and it was estimated that aspartate causes a decrease of the dimer self-association constant by a factor of around 100 (Milligan and Koshland, 1993). These data have prompted the hypothesis that ligand induced receptor dimerization is a prerequisite for signaling (Stock, 1996). Using AUC techniques we have assessed this issue for PcaY_PP. In the absence of ligands, sedimentation velocity studies showed that the protein is present as a virtual species formed by rapidly interchanging monomers and dimers. Multi-speed and multiwavelength sedimentation equilibrium experiments have resulted in the calculation of the dimer association constant of $57.5 \mu \mathrm{M}$. In the presence of ligands, the protein was exclusively present as dimer, confirming the data obtained for Tar-LBD. Ligand induced LBD-dimer stabilization has also been observed for the $4 \mathrm{HB}$ domain containing receptor $\mathrm{CtpH}$ (Rico-Jimenez et al., 2016) as well as for receptors containing a helical bimodular domain (Lacal et al., 2010; Martin-Mora et al., 2016). Inspection of four-helix bundle and helical bimodular domain structures revealed the molecular basis for this stabilization since ligands bind at the dimer interface and amino acids from both monomers establish direct contacts with bound ligand (Milburn et al., 1991; Pineda-Molina et al., 2012). This, however, does not appear to be the case for all chemoreceptor domains since, for example, dCACHE domains were found to be monomeric in solution in the presence and absence of ligand (Rico-Jimenez et al., 2013) and structural information showed that amino acids from only one monomer establish contacts with the bound ligand (Liu et al., 2015). Significant progress has been made in the chemoreceptor identification by high-throughput based ligand screening of recombinant LBDs (McKellar et al., 2015; Fernandez et al., 2016). The monomer-dimer issue is central to the success of this approach since, in the case of four-helix bundle and helical bimodular domains, it has to be assured that at least a fraction of the protein is in a dimeric state.

\section{AUTHOR CONTRIBUTIONS}

TK conceived and coordinated the study and wrote the paper. MF and MM designed, performed and analyzed the experiments shown in Figures 1, 2, 5-7. AO designed, performed and analyzed the experiments shown in Figures 3, 4. All authors reviewed the results and approved the final version of the manuscript.

\section{FUNDING}

We acknowledge financial support from FEDER funds and Fondo Social Europeo through grants from the Junta de Andalucía (grant CVI-7335) and the Spanish Ministry for Economy and Competitiveness (grants BIO2013-42297 and BIO2016-76779-P). MM was supported by the Spanish Ministry of Economy and Competitiveness Postdoctoral Research Program, Juan de la Cierva (JCI-2012-11815).

\section{SUPPLEMENTARY MATERIAL}

The Supplementary Material for this article can be found online at: http://journal.frontiersin.org/article/10.3389/fmicb. 2017.00990/full\#supplementary-material 


\section{REFERENCES}

Abril, M. A., Michan, C., Timmis, K. N., and Ramos, J. L. (1989). Regulator and enzyme specificities of the TOL plasmid-encoded upper pathway for degradation of aromatic hydrocarbons and expansion of the substrate range of the pathway. J. Bacteriol. 171, 6782-6790. doi: 10.1128/jb.171.12.6782-6790. 1989

Bagdasarian, M., Lurz, R., Ruckert, B., Franklin, F. C., Bagdasarian, M. M., Frey, J., et al. (1981). Specific-purpose plasmid cloning vectors. II. Broad host range, high copy number, RSF1010-derived vectors, and a host-vector system for gene cloning in Pseudomonas. Gene 16, 237-247. doi: 10.1016/0378-1119(81) 90080-9

Belda, E., Van Heck, R. G., Lopez-Sanchez, M. J., Cruveiller, S., Barbe, V., Fraser, C., et al. (2016). The revisited genome of Pseudomonas putida KT2440 enlightens its value as a robust metabolic chassis. Environ. Microbiol. 18, 3403-3424. doi: $10.1111 / 1462-2920.13230$

Bentley, R. (1990). The shikimate pathway-a metabolic tree with many branches. Crit. Rev. Biochem. Mol. Biol. 25, 307-384. doi: 10.3109/10409239009090615

Bhatia, A., Bharti, S. K., Tripathi, T., Mishra, A., Sidhu, O. P., Roy, R., et al. (2015). Metabolic profiling of Commiphora wightii (guggul) reveals a potential source for pharmaceuticals and nutraceuticals. Phytochemistry 110, 29-36. doi: 10.1016/j.phytochem.2014.12.016

Bowsher, A. W., Ali, R., Harding, S. A., Tsai, C. J., and Donovan, L. A. (2016). Evolutionary divergences in root exudate composition among ecologicallycontrasting helianthus species. PLoS ONE 11:e0148280. doi: 10.1371/journal. pone. 0148280

Busch, A., Lacal, J., Martos, A., Ramos, J. L., and Krell, T. (2007). Bacterial sensor kinase TodS interacts with agonistic and antagonistic signals. Proc. Natl. Acad. Sci. U.S.A. 104, 13774-13779. doi: 10.1073/pnas.0701547104

Corral-Lugo, A., De La Torre, J., Matilla, M. A., Fernandez, M., Morel, B., EspinosaUrgel, M., et al. (2016). Assessment of the contribution of chemoreceptor-based signaling to biofilm formation. Environ. Microbiol. 18, 3355-3372. doi: 10.1111/ $1462-2920.13170$

Cserzo, M., Wallin, E., Simon, I., Von Heijne, G., and Elofsson, A. (1997). Prediction of transmembrane alpha-helices in prokaryotic membrane proteins: the dense alignment surface method. Protein Eng. 10, 673-676. doi: 10.1093/ protein/10.6.673

Duque, E., Molina-Henares, A. J., De La Torre, J., Molina-Henares, M. A., Del Castillo, T., Lam, J., et al. (2007). "Towards a genome-wide mutant library of Pseudomonas putida strain KT2440," in Pseudomonas: A Model System in Biology, eds J. L. Ramos and A. Filloux (Dordrecht: Springer), 227-251. doi: 10.1007/978-1-4020-6097-7_8

Espinosa-Urgel, M., Kolter, R., and Ramos, J. L. (2002). Root colonization by Pseudomonas putida: love at first sight. Microbiology 148, 341-343. doi: 10.1099/ 00221287-148-2-341

Espinosa-Urgel, M., and Ramos, J. L. (2004). Cell density-dependent gene contributes to efficient seed colonization by Pseudomonas putida KT2440. Appl. Environ. Microbiol. 70, 5190-5198. doi: 10.1128/AEM.70.9.5190-5198.2004

Fernandez, M., Morel, B., Corral-Lugo, A., and Krell, T. (2016). Identification of a chemoreceptor that specifically mediates chemotaxis toward metabolizable purine derivatives. Mol. Microbiol. 99, 34-42. doi: 10.1111/mmi.13215

Galperin, M. Y. (2005). A census of membrane-bound and intracellular signal transduction proteins in bacteria: bacterial IQ, extroverts and introverts. $B M C$ Microbiol. 5:35. doi: 10.1186/1471-2180-5-35

Garcia, V., Reyes-Darias, J. A., Martin-Mora, D., Morel, B., Matilla, M. A., and Krell, T. (2015). Identification of a Chemoreceptor for C2 and C3 Carboxylic Acids. Appl. Environ. Microbiol. 81, 5449-5457. doi: 10.1128/AEM.01529-15

Garcia-Fontana, C., Reyes-Darias, J. A., Munoz-Martinez, F., Alfonso, C., Morel, B., Ramos, J. L., et al. (2013). High specificity in CheR methyltransferase function: CheR2 of Pseudomonas putida is essential for chemotaxis, whereas CheR1 is involved in biofilm formation. J. Biol. Chem. 288, 18987-18999. doi: $10.1074 /$ jbc.M113.472605

Hazelbauer, G. L., Falke, J. J., and Parkinson, J. S. (2008). Bacterial chemoreceptors: high-performance signaling in networked arrays. Trends Biochem. Sci. 33, 9-19. doi: 10.1016/j.tibs.2007.09.014

Hickman, J. W., Tifrea, D. F., and Harwood, C. S. (2005). A chemosensory system that regulates biofilm formation through modulation of cyclic diguanylate levels. Proc. Natl. Acad. Sci. U.S.A. 102, 14422-14427. doi: 10.1073/pnas. 0507170102

Huang, Z., Ni, B., Jiang, C. Y., Wu, Y. F., He, Y. Z., Parales, R. E., et al. (2016). Direct sensing and signal transduction during bacterial chemotaxis toward aromatic compounds in Comamonas testosteroni. Mol. Microbiol. 101, 224-237. doi: $10.1111 / \mathrm{mmi} .13385$

Irwin, J. J., Sterling, T., Mysinger, M. M., Bolstad, E. S., and Coleman, R. G. (2012). ZINC: a free tool to discover chemistry for biology. J. Chem .Inf Model 52, 1757-1768. doi: 10.1021/ci3001277

Jeong, H., Barbe, V., Lee, C. H., Vallenet, D., Yu, D. S., Choi, S. H., et al. (2009). Genome sequences of Escherichia coli B strains REL606 and BL21(DE3). J. Mol. Biol. 394, 644-652. doi: 10.1016/j.jmb.2009.09.052

Kanehisa, M., Furumichi, M., Tanabe, M., Sato, Y., and Morishima, K. (2017). KEGG: new perspectives on genomes, pathways, diseases and drugs. Nucleic Acids Res. 45, D353-D361. doi: 10.1093/nar/gkw1092

Khorassani, R., Hettwer, U., Ratzinger, A., Steingrobe, B., Karlovsky, P., and Claassen, N. (2011). Citramalic acid and salicylic acid in sugar beet root exudates solubilize soil phosphorus. BMC Plant Biol. 11:121. doi: 10.1186/14712229-11-121

Kovach, M. E., Elzer, P. H., Hill, D. S., Robertson, G. T., Farris, M. A., Roop, R. M. II, et al. (1995). Four new derivatives of the broad-host-range cloning vector pBBR1MCS, carrying different antibiotic-resistance cassettes. Gene 166, 175-176. doi: 10.1016/0378-1119(95)00584-1

Lacal, J., Alfonso, C., Liu, X., Parales, R. E., Morel, B., Conejero-Lara, F., et al. (2010). Identification of a chemoreceptor for tricarboxylic acid cycle intermediates: differential chemotactic response towards receptor ligands. J. Biol. Chem. 285, 23126-23136. doi: 10.1074/jbc.M110.110403

Lacal, J., Busch, A., Guazzaroni, M. E., Krell, T., and Ramos, J. L. (2006). The TodSTodT two-component regulatory system recognizes a wide range of effectors and works with DNA-bending proteins. Proc. Natl. Acad. Sci. U.S.A. 103, 8191-8196. doi: 10.1073/pnas.0602902103

Lacal, J., Munoz-Martinez, F., Reyes-Darias, J. A., Duque, E., Matilla, M., Segura, A., et al. (2011). Bacterial chemotaxis towards aromatic hydrocarbons in Pseudomonas. Environ. Microbiol. 13, 1733-1744. doi: 10.1111/j.1462-2920. 2011.02493.x

Laub, M. T., and Goulian, M. (2007). Specificity in two-component signal transduction pathways. Annu. Rev. Genet. 41, 121-145. doi: 10.1146/annurev. genet.41.042007.170548

Laue, T. M., Shah, B. D., Ridgeway, T. M., and Pelletier, S. L. (1992). "Computeraided interpretation of analytical sedimentation data for proteins," in Analytical Ultracentrifugation in Biochemistry and Polymer Science, eds S. Harding, A Rowe, and J Horton (Cambridge: Royal Society of Chemistry), 90-125.

Lehmann, M. M., Wegener, F., Werner, R. A., and Werner, C. (2016). Diel variations in carbon isotopic composition and concentration of organic acids and their impact on plant dark respiration in different species. Plant Biol. (Stuttg.) 18, 776-784. doi: 10.1111/plb.12464

Liu, Y. C., Machuca, M. A., Beckham, S. A., Gunzburg, M. J., and Roujeinikova, A. (2015). Structural basis for amino-acid recognition and transmembrane signalling by tandem Per-Arnt-Sim (tandem PAS) chemoreceptor sensory domains. Acta Crystallogr. D. Biol. Crystallogr. 71, 2127-2136. doi: 10.1107/ S139900471501384X

Luu, R. A., Kootstra, J. D., Nesteryuk, V., Brunton, C. N., Parales, J. V., Ditty, J. L., et al. (2015). Integration of chemotaxis, transport and catabolism in Pseudomonas putida and identification of the aromatic acid chemoreceptor PcaY. Mol. Microbiol. 96, 134-147. doi: 10.1111/mmi.12929

Martin-Mora, D., Reyes-Darias, J. A., Ortega, A., Corral-Lugo, A., Matilla, M. A., and Krell, T. (2016). McpQ is a specific citrate chemoreceptor that responds preferentially to citrate/metal ion complexes. Environ. Microbiol. 18, 3284-3295. doi: 10.1111/1462-2920.13030

Matilla, M. A., Espinosa-Urgel, M., Rodriguez-Herva, J. J., Ramos, J. L., and Ramos-Gonzalez, M. I. (2007). Genomic analysis reveals the major driving forces of bacterial life in the rhizosphere. Genome Biol. 8:R179. doi: 10.1186/ gb-2007-8-9-r179

Matilla, M. A., and Krell, T. (2017). Chemoreceptor-based signal sensing. Curr. Opin. Biotechnol. 45, 8-14. doi: 10.1016/j.copbio.2016.11.021

Matilla, M. A., Ramos, J. L., Bakker, P. A., Doornbos, R., Badri, D. V., Vivanco, J. M., et al. (2010). Pseudomonas putida KT2440 causes induced systemic resistance 
and changes in Arabidopsis root exudation. Environ. Microbiol. Rep. 2, 381-388. doi: 10.1111/j.1758-2229.2009.00091.x

McKellar, J. L., Minnell, J. J., and Gerth, M. L. (2015). A high-throughput screen for ligand binding reveals the specificities of three amino acid chemoreceptors from Pseudomonas syringae pv. actinidiae. Mol. Microbiol. 96, 694-707. doi: $10.1111 / \mathrm{mmi} .12964$

Milburn, M. V., Prive, G. G., Milligan, D. L., Scott, W. G., Yeh, J., Jancarik, J., et al. (1991). Three-dimensional structures of the ligand-binding domain of the bacterial aspartate receptor with and without a ligand. Science 254, 1342-1347. doi: $10.1126 /$ science. 1660187

Milligan, D. L., and Koshland, D. E. Jr. (1993). Purification and characterization of the periplasmic domain of the aspartate chemoreceptor. J. Biol. Chem. 268, 19991-19997.

Molina, L., Ramos, C., Duque, E., Ronchel, M. C., Garcia, J. M., Wyke, L., et al. (2000). Survival of Pseudomonas putida KT2440 in soil and in the rhizosphere of plants under greenhouse and environmental conditions. Soil Biol. Biochem. 32, 315-321. doi: 10.1016/S0038-0717(99)00156-X

Nakazawa, T. (2002). Travels of a Pseudomonas, from Japan around the world. Environ. Microbiol. 4, 782-786. doi: 10.1046/j.1462-2920.2002.00310.x

Neal, A. L., Ahmad, S., Gordon-Weeks, R., and Ton, J. (2012). Benzoxazinoids in root exudates of maize attract Pseudomonas putida to the rhizosphere. PLoS ONE 7:e35498. doi: 10.1371/journal.pone.0035498

Ni, B., Huang, Z., Fan, Z., Jiang, C. Y., and Liu, S. J. (2013a). Comamonas testosteroni uses a chemoreceptor for tricarboxylic acid cycle intermediates to trigger chemotactic responses towards aromatic compounds. Mol. Microbiol. 90, 813-823. doi: 10.1111/mmi.12400

Ni, B., Huang, Z., Wu, Y. F., Fan, Z., Jiang, C. Y., and Liu, S. J. (2015). A novel chemoreceptor MCP2983 from Comamonas testosteroni specifically binds to cis-aconitate and triggers chemotaxis towards diverse organic compounds. Appl. Microbiol. Biotechnol. 99, 2773-2781. doi: 10.1007/s00253-014-6216-3

Ni, B., Zhang, Y., Chen, D. W., Wang, B. J., and Liu, S. J. (2013b). Assimilation of aromatic compounds by Comamonas testosteroni: characterization and spreadability of protocatechuate 4,5-cleavage pathway in bacteria. Appl. Microbiol. Biotechnol. 97, 6031-6041. doi: 10.1007/s00253-012-4402-8

Parales, R. E., Luu, R. A., Chen, G. Y., Liu, X., Wu, V., Lin, P., et al. (2013). Pseudomonas putida F1 has multiple chemoreceptors with overlapping specificity for organic acids. Microbiology 159, 1086-1096. doi: 10.1099/mic.0. 065698-0

Parkinson, J. S., Hazelbauer, G. L., and Falke, J. J. (2015). Signaling and sensory adaptation in Escherichia coli chemoreceptors: 2015 update. Trends Microbiol. 23, 257-266. doi: 10.1016/j.tim.2015.03.003

Persat, A., Inclan, Y. F., Engel, J. N., Stone, H. A., and Gitai, Z. (2015). Type IV pili mechanochemically regulate virulence factors in Pseudomonas aeruginosa. Proc. Natl. Acad. Sci. U.S.A. 112, 7563-7568. doi: 10.1073/pnas.1502025112

Pineda-Molina, E., Reyes-Darias, J. A., Lacal, J., Ramos, J. L., Garcia-Ruiz, J. M., Gavira, J. A., et al. (2012). Evidence for chemoreceptors with bimodular ligandbinding regions harboring two signal-binding sites. Proc. Natl. Acad. Sci. U.S.A. 109, 18926-18931. doi: 10.1073/pnas.1201400109

Pizarro-Tobias, P., Fernandez, M., Niqui, J. L., Solano, J., Duque, E., Ramos, J. L., et al. (2015). Restoration of a Mediterranean forest after a fire: bioremediation and rhizoremediation field-scale trial. Microb. Biotechnol. 8, 77-92. doi: 10. 1111/1751-7915.12138

Regenhardt, D., Heuer, H., Heim, S., Fernandez, D. U., Strompl, C., Moore, E. R., et al. (2002). Pedigree and taxonomic credentials of Pseudomonas putida strain KT2440. Environ. Microbiol. 4, 912-915. doi: 10.1046/j.1462-2920.2002. 00368.x

Reyes-Darias, J. A., Garcia, V., Rico-Jimenez, M., Corral-Lugo, A., Lesouhaitier, O., Juarez-Hernandez, D., et al. (2015a). Specific gamma-aminobutyrate chemotaxis in pseudomonads with different lifestyle. Mol. Microbiol. 97, 488-501. doi: 10.1111/mmi.13045

Reyes-Darias, J. A., Yang, Y., Sourjik, V., and Krell, T. (2015b). Correlation between signal input and output in PctA and PctB amino acid chemoreceptor of Pseudomonas aeruginosa. Mol. Microbiol. 96, 513-525. doi: 10.1111/mmi.12953
Rico-Jimenez, M., Munoz-Martinez, F., Garcia-Fontana, C., Fernandez, M., Morel, B., Ortega, A., et al. (2013). Paralogous chemoreceptors mediate chemotaxis towards protein amino acids and the non-protein amino acid gamma-aminobutyrate (GABA). Mol. Microbiol. 88, 1230-1243. doi: 10.1111/ mmi.12255

Rico-Jimenez, M., Reyes-Darias, J. A., Ortega, A., Diez Pena, A. I., Morel, B., and Krell, T. (2016). Two different mechanisms mediate chemotaxis to inorganic phosphate in Pseudomonas aeruginosa. Sci. Rep. 6:28967. doi: 10. 1038/srep28967

Sarand, I., Osterberg, S., Holmqvist, S., Holmfeldt, P., Skarfstad, E., Parales, R. E., et al. (2008). Metabolism-dependent taxis towards (methyl)phenols is coupled through the most abundant of three polar localized Aer-like proteins of Pseudomonas putida. Environ. Microbiol. 10, 1320-1334. doi: 10.1111/j.14622920.2007.01546.x

Scharf, B. E., Hynes, M. F., and Alexandre, G. M. (2016). Chemotaxis signaling systems in model beneficial plant-bacteria associations. Plant Mol. Biol. 90, 549-559. doi: 10.1007/s11103-016-0432-4

Schuck, P. (2000). Size-distribution analysis of macromolecules by sedimentation velocity ultracentrifugation and lamm equation modeling. Biophys. J. 78, 1606-1619. doi: 10.1016/S0006-3495(00)76713-0

Stock, J. (1996). Receptor signaling: dimerization and beyond. Curr. Biol. 6, 825-827. doi: 10.1016/S0960-9822(02)00605-X

Swinnen, I. A., Bernaerts, K., Dens, E. J., Geeraerd, A. H., and Van Impe, J. F. (2004). Predictive modelling of the microbial lag phase: a review. Int. J. Food Microbiol. 94, 137-159. doi: 10.1016/j.ijfoodmicro.2004.01.006

Tawaraya, K., Horie, R., Saito, S., Wagatsuma, T., Saito, K., and Oikawa, A. (2014). Metabolite profiling of root exudates of common bean under phosphorus deficiency. Metabolites 4, 599-611. doi: 10.3390/metabo4030599

Ulrich, L. E., and Zhulin, I. B. (2005). Four-helix bundle: a ubiquitous sensory module in prokaryotic signal transduction. Bioinformatics 21(Suppl. 3), iii45-iii48. doi: 10.1093/bioinformatics/bti1204

Vistica, J., Dam, J., Balbo, A., Yikilmaz, E., Mariuzza, R. A., Rouault, T. A., et al. (2004). Sedimentation equilibrium analysis of protein interactions with global implicit mass conservation constraints and systematic noise decomposition. Anal. Biochem. 326, 234-256. doi: 10.1016/j.ab.2003.12.014

Woodcock, D. M., Crowther, P. J., Doherty, J., Jefferson, S., Decruz, E., Noyer-Weidner, M., et al. (1989). Quantitative evaluation of Escherichia coli host strains for tolerance to cytosine methylation in plasmid and phage recombinants. Nucleic Acids Res. 17, 3469-3478. doi: 10.1093/nar/17.9.3469

Wuichet, K., and Zhulin, I. B. (2010). Origins and diversification of a complex signal transduction system in prokaryotes. Sci. Signal. 3:ra50. doi: 10.1126/ scisignal.2000724

Yang, Y., M, Pollard A, Höfler, C., Poschet, G., Wirtz, M., Hell, R., et al. (2015). Relation between chemotaxis and consumption of amino acids in bacteria. Mol. Microbiol. 96, 1272-1282. doi: 10.1111/mmi.13006

Zusman, D. R., Scott, A. E., Yang, Z., and Kirby, J. R. (2007). Chemosensory pathways, motility and development in Myxococcus xanthus. Nat. Rev. Microbiol. 5, 862-872. doi: 10.1038/nrmicro1770

Conflict of Interest Statement: The authors declare that the research was conducted in the absence of any commercial or financial relationships that could be construed as a potential conflict of interest.

The reviewer FAP and handling Editor declared their shared affiliation, and the handling Editor states that the process nevertheless met the standards of a fair and objective review.

Copyright (c) 2017 Fernández, Matilla, Ortega and Krell. This is an open-access article distributed under the terms of the Creative Commons Attribution License (CC BY). The use, distribution or reproduction in other forums is permitted, provided the original author(s) or licensor are credited and that the original publication in this journal is cited, in accordance with accepted academic practice. No use, distribution or reproduction is permitted which does not comply with these terms. 